\title{
Operations on Intuitionistic Fuzzy Hypergraphs
}

\author{
R.Parvathi \\ Department of Mathematics \\ Vellalar College for Women \\ Erode, Tamilnadu, India.
}

\author{
S.Thilagavathi \\ Department of Mathematics \\ Vellalar College for Women \\ Erode, Tamilnadu, India.
}

\author{
M.G.Karunambigai \\ Department of Mathematics \\ Sri Vasavi College, Erode \\ Tamilnadu, India.
}

\begin{abstract}
Hypergraph is a graph in which an edge can connect more than two vertices. Hypergraphs can be applied to analyze architecture structures and to represent system partitions. The concept of hypergraphs was extended to fuzzy hypergraph. In this paper, we extend the concepts of fuzzy hypergraphs into that of intuitionistic fuzzy hypergraphs. Based on the definition of intuitionistic fuzzy graph, operations like complement, join, union, intersection, ringsum, cartesian product, composition are defined for intuitionistic fuzzy graphs. The authors further proposed to apply these operations in clustering techniques.
\end{abstract}

\section{Keywords}

Intuitionistic fuzzy hypergraph (IFHG), Complement, Union, Join, Intersection, Ringsum, Cartesian product, Composition.

\section{INTRODUCTION}

Hypergraph theory, originally developed by C.Berge in 1960 , is a generalization of graph theory. The concept of hypergraphs can model more general types of relations than binary relations. The notion of hypergraphs has been extended in fuzzy theory and the concept of fuzzy hypergraphs was proposed by Lee-Kwang and S.M.Chen. The concept of an intuitionistic fuzzy graph (IFG) was introduced by Atanassov $[1,2,3,4]$. The authors have already introduced the concept of intuitionistic fuzzy hypergraph [7]. Operations on IFGs have also been analyzed by the authors [5,6]. Akram [8] applied the concepts in [7] to a real-life problem with a numerical example.

\section{PRELIMINARIES}

\section{Definition 2.1}

An intuitionistic fuzzy graph (IFG) is of the $G=\langle V, E\rangle$ where (i) $V=\left\{v_{1}, v_{2}, \ldots, v_{n}\right\} \quad$ such that $\mu_{1}: V \rightarrow[0,1]$ and $\gamma_{1}: V \rightarrow[0,1]$ denote the degree of membership and non-membership of the element $v_{i} \in V$ respectively and

$$
0 \leq \mu_{1}\left(v_{i}\right)+\gamma_{1}\left(v_{i}\right) \leq 1
$$

for every $v_{i} \in V,(i=1,2, \ldots, \mathrm{n})$, (ii) $E \subseteq V \times V$ where $\mu_{2}: V \times V \rightarrow[0,1]$ and $\gamma_{2}: V \times V \rightarrow[0,1]$ are such that

$$
\begin{aligned}
& \mu_{2}\left(v_{i}, v_{j}\right) \leq \mu_{1}\left(v_{i}\right) \cdot \mu_{1}\left(v_{j}\right) \\
& \gamma_{2}\left(v_{i}, v_{j}\right) \leq \gamma_{1}\left(v_{i}\right) \cdot \gamma_{1}\left(v_{j}\right)
\end{aligned}
$$

and $0 \leq \mu_{2}\left(v_{i}, v_{j}\right)+\gamma_{2}\left(v_{i}, v_{j}\right) \leq 1$

for every $\left(v_{i}, v_{j}\right) \in E$.

\section{Definition 2.2}

An IFHG $H$ is an ordered pair $H=\langle V, E\rangle$ where (i) $\quad V=\left\{v_{1}, v_{2}, \ldots, v_{n}\right\}, \quad$ a finite set of vertices (ii) $E=\left\{E_{1}, E_{2}, \ldots, E_{n}\right\}$, a family of intuitionistic fuzzy subsets of $V$. . (iii) $E_{j}=\left\{\left(v_{i}, \mu_{j}\left(v_{i}\right), \gamma_{j}\left(v_{i}\right)\right): \mu_{j}\left(v_{i}\right), \gamma_{j}\left(v_{i}\right) \geq 0\right.$ and $0 \leq \mu_{j}\left(v_{i}\right)+\gamma_{j}\left(v_{i}\right) \leq 1, j=1,2, \ldots, m$ (iv) $E_{j} \neq \varphi \quad j=$ $1,2, \ldots, m$ (v) $\mathrm{U}_{j} \operatorname{supp}\left(E_{j}\right)=V, j=1,2, \ldots, m$

Here the edges $E_{j}$ are IFSs. $\mu_{j}\left(x_{i}\right)$ and $\gamma_{j}\left(x_{i}\right)$ denote the degree of membership and non-membership of the vertex $v_{i}$ to edge $E_{j}$. Thus, the elements of the incidence matrix of IFHG are of the form $\left(a_{i j}, \mu_{j}\left(x_{i}\right), \gamma_{j}\left(x_{i}\right)\right)$. The sets $V$ and $E$ are crisp sets.

\section{Notations}

The triple $\left\langle v_{i}, \mu_{1 i}, \gamma_{1 i}\right\rangle$ denotes the degree of membership and non-membership of the vertex $v_{i}$. The triple $\left\langle e_{i j}, \mu_{2 i j}, \gamma_{2 i j}\right\rangle$ denotes the degree of membership and non-membership of the edge $e_{i j}=\left(v_{i}, v_{j}\right)$ on $V$. That is,

$$
\mu_{1 i}=\mu_{1}\left(v_{i}\right), \gamma_{1 i}=\gamma_{1}\left(v_{i}\right) \text { and } \quad \mu_{2 i j}=\mu_{2}\left(v_{i}, v_{j}\right), \gamma_{2 i j}=\gamma_{2}\left(v_{i}, v_{j}\right) \text {. }
$$

\section{Note 2.3}

(i) When $\mu_{2 i j}=\gamma_{2 i j}=0$, for some $i$ and $j$, then there is no edge between $v_{i}$ and $v_{j}$. Otherwise there exists edge between $v_{i}$ and $v_{j}$. (ii) If one of the inequalities (1) or (2) or (3) or (4) is not satisfied, then $G$ is not an IFHG. 


\section{Definition 2.4}

An IFHG,$G=\langle V, E\rangle$ is said to be a semi - $\mu$ strong IFHG if $\mu_{2 i j}=\mu_{1 i} \cdot \mu_{1 j}$ for every $i$ and $j$.

\section{Definition 2.5}

An IFHG,$G=\langle V, E\rangle$ is said to be a semi $-\gamma$ strong IFHG if $\gamma_{2 i j}=\gamma_{1 i} \cdot \gamma_{1 j}$ for every $i$ and $j$.

\section{Definition 2.6}

An IFHG $G=\langle V, E\rangle$ is said to be a strong IFHG if $\mu_{2 i j}=\mu_{1 i} \cdot \mu_{1 j}$ and $\gamma_{2 i j}=\gamma_{1 i} \cdot \gamma_{1 j}$ for all $\left(v_{i}, v_{j}\right) \in E$.

\section{Definition 2.7}

An IFHG,$G=\langle V, E\rangle$ is said to be a complete $-\mu$ strong

IFHG if $\mu_{2 i j}=\mu_{1 i} \cdot \mu_{1 j}$ and $\gamma_{2 i j}<\gamma_{1 i} \cdot \gamma_{1 j}$

\section{Definition 2.8}

An IFHG,$G=\langle V, E\rangle$ is said to be a complete $-\gamma$ strong IFHG if $\mu_{2 i j}<\mu_{1 i} \cdot \mu_{1 j}$ and $\gamma_{2 i j}=\gamma_{1 i} \cdot \gamma_{1 j}$ for all $i$ and $j$.

\section{Definition 2.9}

An IFHG , $G=\langle V, E\rangle$ is said to be a complete IFHG if $\mu_{2 i j}=\mu_{1 i} \cdot \mu_{1 j}$ and $\gamma_{2 i j}=\gamma_{1 i} \cdot \gamma_{1 j}$, for every $\left(v_{i}, v_{j}\right) \in V$.

\section{COMPLEMENT OF AN IFHG}

\section{Definition 3.1}

The complement of an IFHG $G=\langle V, E\rangle$ is an IFHG, $\bar{G}=\langle\bar{V}, \bar{E}\rangle$, where

- $\bar{V}=V$

- $\overline{\mu_{1 i}}=\mu_{1 i}$ and $\overline{\gamma_{1 i}}=\gamma_{1 i}$, for all $i=1,2, \ldots, \mathrm{n}$.

- $\overline{\mu_{2 i j}}=\left(\mu_{1 i} \cdot \mu_{1 j}\right)-\mu_{2 i j}$ and $\overline{\gamma_{2 i j}}=\left(\gamma_{1 i} \cdot \gamma_{1 j}\right)-\gamma_{2 i j}$ for all $i$, $j=1,2, \ldots, \mathrm{n}$.

Example 3.2

Let $V=\left\{v_{1}, v_{2}, v_{3}, v_{4}\right\}$ and $E=\left\{e_{1}, e_{2}\right\}$

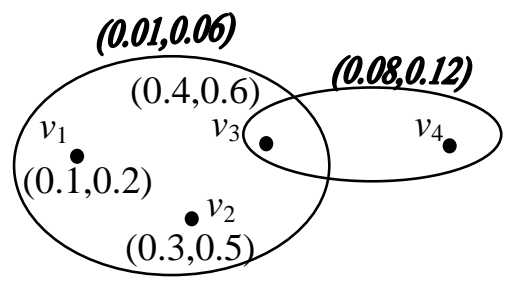

$(0.2,0.2)$

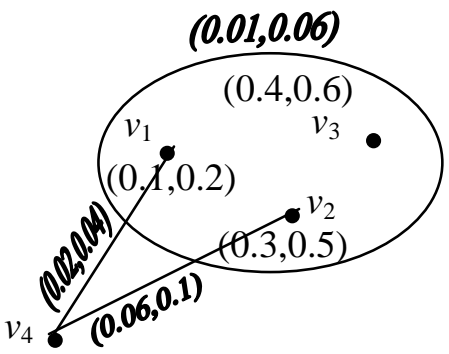

$(0.2,0.2)$

Fig 1: An Intuitionistic fuzzy hypergraph and its complement

\section{Note 3.3}

One can easily verify that $\bar{G}=G$.

\section{Proposition 3.4}

(i) The complement of a complete $\mu$ - strong IFHG is a complete IFHG.

(ii) The complement of a complete $\gamma$ - strong IFHG is a complete IFHG.

\section{Proof}

(i) Let $G=\langle V, E\rangle$ be a complete $\mu$-strong IFHG. Therefore, $\mu_{2 i j}=\mu_{1 i} \cdot \mu_{1 j}$ and $\gamma_{2 i j}<\gamma_{1 i} \cdot \gamma_{1 j}$ for all $i$ and $j$.

To prove that either (a) $\bar{\mu}_{2 i j}>0$ or $\bar{\gamma}_{2 i j}>0$, (b) $\bar{\mu}_{2 i j}=0$ or $\bar{\gamma}_{2 i j}>0$.
That is, $\bar{\mu}_{2 i j}=\left(\mu_{1 i} \cdot \mu_{1 j}\right)-\mu_{2 i j}$

$$
=0 \text { if } \mu_{2 i j}>0
$$

$$
=\mu_{1 i} \text { if } \mu_{2 i j}=0 \text { and }
$$

$\bar{\gamma}_{2 i j}=\left(\gamma_{1 i} \cdot \gamma_{1 j}\right)-\gamma_{2 i j}>0$ for every $i$ and $j$, since $G$ is a complete $\mu$-strong IFHG. (ii) Proof of (ii) is the same as proof of (i)

\section{Definition 3.5}

Consider the two IFHGs $G_{1}=\left\langle V_{1}, E_{1}\right\rangle$ and $G_{2}=\left\langle V_{2}, E_{2}\right\rangle$. An isomorphism between two IFHGs $G_{1}$ and $G_{2}$, denoted by $G_{1} \cong G_{2}$, is a bijective map $h: V_{1} \rightarrow V_{2}$ which satisfies $\mu_{1}\left(v_{i}\right)=\mu_{1}^{\prime}\left(h\left(v_{i}\right)\right) ; \gamma_{1}\left(v_{i}\right)=\gamma_{1}^{\prime}\left(h\left(v_{i}\right)\right)$ and 
$\mu_{2}\left(v_{i}, v_{j}\right)=\mu_{2}^{\prime}\left(h\left(v_{i}\right), h\left(v_{j}\right)\right) ; \gamma_{2}\left(v_{i}, v_{j}\right)=\gamma_{2}^{\prime}\left(h\left(v_{i}\right), h\left(v_{j}\right)\right.$ for

$\operatorname{every}\left(v_{l}, v_{j}\right) \in V$.

\section{Definition 3.6}

An IFHG $G$ is self-complementary if $G$ is isomorphic to $\bar{G}$. Symbolically, $G_{1} \cong G_{2}$.

\section{Theorem 3.7}

Let $G=\langle V, E\rangle$ be a self-complementary IFHG. Then, there exists an isomorphism $h: V \rightarrow V$ such that $\overline{\mu_{1}}\left(v_{i}\right)=\mu_{1 i} ; \bar{\gamma}_{1}\left(v_{i}\right)=\gamma_{1 i}, \quad$ for $\quad$ every $\quad v_{i} \in V \quad$ and $\overline{\mu_{2}}\left(h\left(v_{i}\right), h\left(v_{j}\right)\right)=\mu_{2 i} ; \quad \overline{\gamma_{2}}\left(h\left(v_{i}\right), h\left(v_{j}\right)\right)=\gamma_{2 i} \quad$ for $\quad$ every $\left(v_{l}, v_{j}\right) \in V$.

Proof

By Definition $3.1, \quad$ we $\overline{\mu_{2}}\left(h\left(v_{i}\right), h\left(v_{j}\right)\right)=\left(\overline{\mu_{1}}\left(h\left(v_{i}\right)\right) \cdot \overline{\mu_{1}}\left(h\left(v_{j}\right)\right)\right)-\mu_{2 i j}$

$\Rightarrow \mu_{2}\left(v_{i}, v_{j}\right)=\mu_{1 i} \cdot \mu_{1 j}-\mu_{2 i j} \Rightarrow \mu_{2 i j}=\mu_{1 i} \cdot \mu_{1 j}-\mu_{2 i j}$

$\Rightarrow \sum_{i \neq j} \mu_{2 i j}=\sum_{i \neq j}\left(\mu_{1 i} \cdot \mu_{1 j}\right)-\sum_{i \neq j} \mu_{2 i j} \Rightarrow 2 \sum_{i \neq j} \mu_{2 i j}=\sum_{i \neq j}\left(\mu_{1 i} \cdot \mu_{1 j}\right)$

$\Rightarrow \sum_{i \neq j} \mu_{2 i j}=\frac{1}{2} \sum_{i \neq j}\left(\mu_{1 i} \cdot \mu_{1 j}\right)$.

and

$\overline{\gamma_{2}}\left(h\left(v_{i}\right), h\left(v_{j}\right)\right)=\left(\overline{\gamma_{1}}\left(h\left(v_{i}\right)\right) \cdot \bar{\gamma}_{1}\left(h\left(v_{j}\right)\right)\right)-\gamma_{2 i j}$

$\Rightarrow \gamma_{2 i j}=\left(\gamma_{1 i} \cdot \gamma_{1 j}\right)-\gamma_{2 i j} \Rightarrow \sum_{i \neq j} \gamma_{2 i j}=\sum_{i \neq j}\left(\gamma_{1 i} \cdot \gamma_{1 j}\right)-\sum_{i \neq j} \gamma_{2 i j}$

$\Rightarrow 2 \sum_{i \neq j} \gamma_{2 i j}=\sum_{i \neq j}\left(\gamma_{1 i} \cdot \gamma_{1 j}\right) \Rightarrow \sum_{i \neq j} \gamma_{2 i j}=\frac{1}{2} \sum_{i \neq j}\left(\gamma_{1 i} \cdot \gamma_{1 j}\right)$.

\section{Remark 3.8}

The condition in the above theorem is not sufficient.

\section{Theorem 3.9}

If $G$ is a strong IFHG, then $\bar{G}$ is also strong.

Proof

Let $u v \in E$. Then

$\overline{\mu_{2}}(u v)=\mu_{1}(u) \cdot \mu_{1}(v)-\mu_{2}(u v)$

$=\mu_{1}(u) \cdot \mu_{1}(v)-\mu_{1}(u) \cdot \mu_{1}(v)$, since $G$ is strong

$=0$ and $\overline{\gamma_{2}}(u v)=\gamma_{1}(u) \cdot \gamma_{1}(v)-\gamma_{2}(u v)=\gamma_{1}(u) \cdot \gamma_{1}(v)-\gamma_{1}(u) \cdot \gamma_{1}(v)$, since $G$ is strong

$=0$.

Let $u v \notin E$. Then $\overline{\mu_{2}}(u v)=\left(\mu_{1}(u) \cdot \mu_{1}(v)\right)-\mu_{2}(u v)=$ $\mu_{1}(u) \cdot \mu_{1}(v)$ and $\overline{\gamma_{2}}(u v)=\gamma_{1}(u) \cdot \gamma_{1}(v)-\gamma_{2}(u v)=\gamma_{1}(u) \cdot \gamma_{1}(v)$.

\section{OPERATIONS ON INTUITIONISTIC FUZZY HYPERGRAPHS}

\section{Definition 4.1}

Let $G_{1}=\left\langle V_{1}, E_{1}\right\rangle$ and $G_{2}=\left\langle V_{2}, E_{2}\right\rangle$ be two IFHGs with $V_{1}$ I $V_{2}=\varphi$. Then the union of $G_{1}$ and $G_{2}$ is an IFHG $G=G_{1} \mathrm{U} G_{2}=\left\langle V_{1} \mathrm{U} V_{2}, E_{1} \mathrm{U} E_{2}\right\rangle$ defined by

$\left(\mu_{1} \mathrm{U} \mu_{1}^{\prime}\right)(v)=\left\{\begin{array}{lll}\mu_{1}(v) & \text { if } & v \in V_{1}-V_{1} \\ \mu_{1}^{\prime}(v) & \text { if } & v \in V_{2}-V_{1}\end{array}\right.$

$\left(\gamma_{1} \mathrm{U} \gamma_{1}^{\prime}\right)(v)=\left\{\begin{array}{lll}\gamma_{1}(v) & \text { if } & v \in V_{1}-V_{2} \\ \gamma_{1}^{\prime}(v) & \text { if } & v \in V_{2}-V_{1}\end{array}\right.$

and $\left(\mu_{2} \mathrm{U} \mu_{2}^{\prime}\right)\left(v_{i} v_{j}\right)=\left\{\begin{array}{lll}\mu_{2 i j} & \text { if } & e_{i j} \in E_{1}-E_{2} \\ \mu_{2 i j}^{\prime} & \text { if } & e_{i j} \in E_{2}-E_{1}\end{array}\right.$

$\left(\gamma_{2} \mathrm{U} \gamma_{2}^{\prime}\right)\left(v_{i} v_{j}\right)= \begin{cases}\gamma_{2 i j} & \text { if } e_{i j} \in E_{1}-E_{2} \\ \gamma_{2 i j}^{\prime} & \text { if } e_{i j} \in E_{2}-E_{1}\end{cases}$

where $\left(\mu_{1}, \gamma_{1}\right)$ and $\left(\mu_{1}^{\prime}, \gamma_{1}^{\prime}\right)$ refer the vertex membership and non-membership of $G_{1}$ and $G_{2}$ respectively; $\left(\mu_{2}, \gamma_{2}\right)$ and $\left(\mu_{2}{ }^{\prime}, \gamma_{2}{ }^{\prime}\right)$ refer the edge membership and non-membership of $G_{1}$ and $G_{2}$ respectively.

\section{Definition 4.2}

The join of two IFHGs $G_{1}$ and $G_{2}$ is an IFHG $G=G_{1}+G_{2}=$ $\left\langle V_{1} \mathrm{U} V_{2}, E_{1} \mathrm{U} E_{2} \mathrm{U} E^{\prime}\right\rangle$ defined by

$$
\begin{gathered}
\left(\mu_{1}+\mu_{1}^{\prime}\right)(v)=\left(\mu_{1} \mathrm{U} \mu_{1}^{\prime}\right)(v) \quad \text { if } v \in V_{1} \mathrm{U} V_{2} \\
\left(\gamma_{1}+\gamma_{1}^{\prime}\right)(v)=\left(\gamma_{1} \mathrm{U} \gamma_{1}^{\prime}\right)(v) \text { if } v \in V_{1} \mathrm{U} V_{2}
\end{gathered}
$$

$\left(\mu_{2}+\mu_{2}^{\prime}\right)\left(v_{i} v_{j}\right)=\left(\mu_{2} \mathrm{U} \mu_{2}^{\prime}\right)\left(v_{i} v_{j}\right)$ if $v_{i} v_{j} \in E_{1} \mathrm{U} E_{2}$

$$
=\left(\mu_{1}\left(v_{i}\right) \cdot \mu_{1}^{\prime}\left(v_{j}\right) \text { if } v_{i} v_{j} \in E^{\prime}\right.
$$

and

$$
\begin{gathered}
\left(\gamma_{2}+\gamma_{2}^{\prime}\right)\left(v_{i} v_{j}\right)=\left(\gamma_{2} \mathrm{U} \gamma_{2}^{\prime}\right)\left(v_{i} v_{j}\right) \quad \text { if } v_{i} v_{j} \in E_{1} \mathrm{U} E_{2} \\
=\left(\gamma_{1}\left(v_{i}\right) \cdot \gamma_{1}^{\prime}\left(v_{j}\right) \text { if } v_{i} v_{j} \in E^{\prime} .\right.
\end{gathered}
$$

\section{Definition 4.3}

Let $G_{1}=\left\langle V_{1}, E_{1}\right\rangle$ and $G_{2}=\left\langle V_{2}, E_{2}\right\rangle$ be two IFHGs. Then the intersection of IFHGs $G_{1}$ and $G_{2}$ denoted by $G_{1}$ I $G_{2}$ is an IFHG defined by $\left(\mu_{1} \mathrm{I} \mu_{1}^{\prime}\right)(v)=\left\{\begin{array}{lll}\mu_{1}(v) & \text { if } & v \in V_{1} \text { and } V_{2} \\ \mu_{1}^{\prime}(v) & \text { if } & v \in V_{2} \text { and } V_{1}\end{array}\right.$

$\left(\gamma_{1} \mathrm{I} \quad \gamma_{1}^{\prime}\right)(v)=\left\{\begin{array}{lll}\gamma_{1}(v) & \text { if } & v \in V_{1} \text { and } V_{2} \\ \gamma_{1}^{\prime}(v) & \text { if } \quad v \in V_{2} \text { and } V_{1}\end{array}\right.$ 
and

$\left(\mu_{2} \mathrm{I} \quad \mu_{2}{ }^{\prime}\right)\left(v_{i} v_{j}\right)=\left\{\begin{array}{lll}\mu_{2 i j} & \text { if } e_{i j} \in E_{1} \text { and } E_{2} \\ \mu_{2 i j}{ }^{\prime} & \text { if } & e_{i j} \in E_{1} \text { and } E_{2}\end{array}\right.$

$\left(\gamma_{2} \mathrm{I} \quad \gamma_{2}^{\prime}\right)\left(v_{i} v_{j}\right)=\left\{\begin{array}{lll}\gamma_{2 i j} & \text { if } e_{i j} \in E_{1} \text { and } E_{2} \\ \gamma_{2 i j}{ }^{\prime} & \text { if } & e_{i j} \in E_{1} \text { and } E_{2}\end{array}\right.$

Definition 4.4

Let $G_{1}=\left\langle V_{1}, E_{1}\right\rangle$ and $G_{2}=\left\langle V_{2}, E_{2}\right\rangle$ be two IFHGs. Then the ringsum of IFHGs $G_{1}$ and $G_{2}$ is an IFHG defined by $G_{1} \oplus G_{2}=\left(G_{1} \mathrm{U} G_{2}\right)-\left(G_{1} \mathrm{I} G_{2}\right)$.
Example 4.5

Let $V_{1}=\left\{v_{1}, v_{2}, v_{3}, v_{4}\right\}$ and $V_{2}=\left\{u_{1}, u_{2}\right\}$ such that $V_{1} \mathrm{I} V_{2}=\varphi$
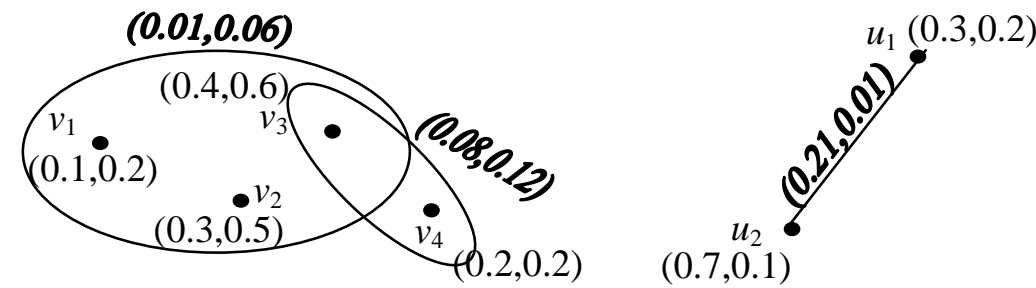

Fig 2: $G_{1}$ and $G_{2}$
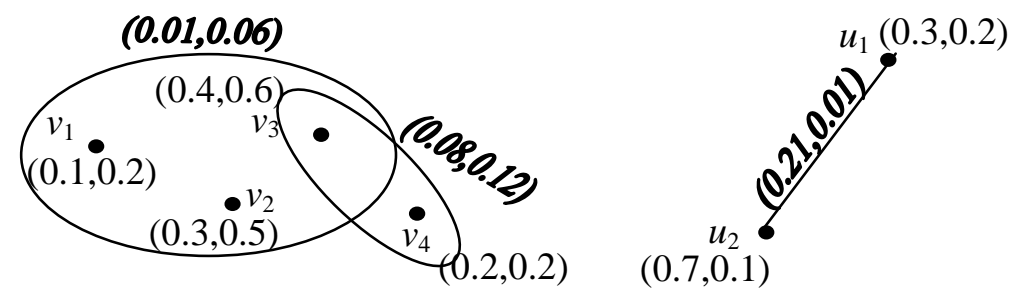

Fig 3: $G_{1} U G_{2}$

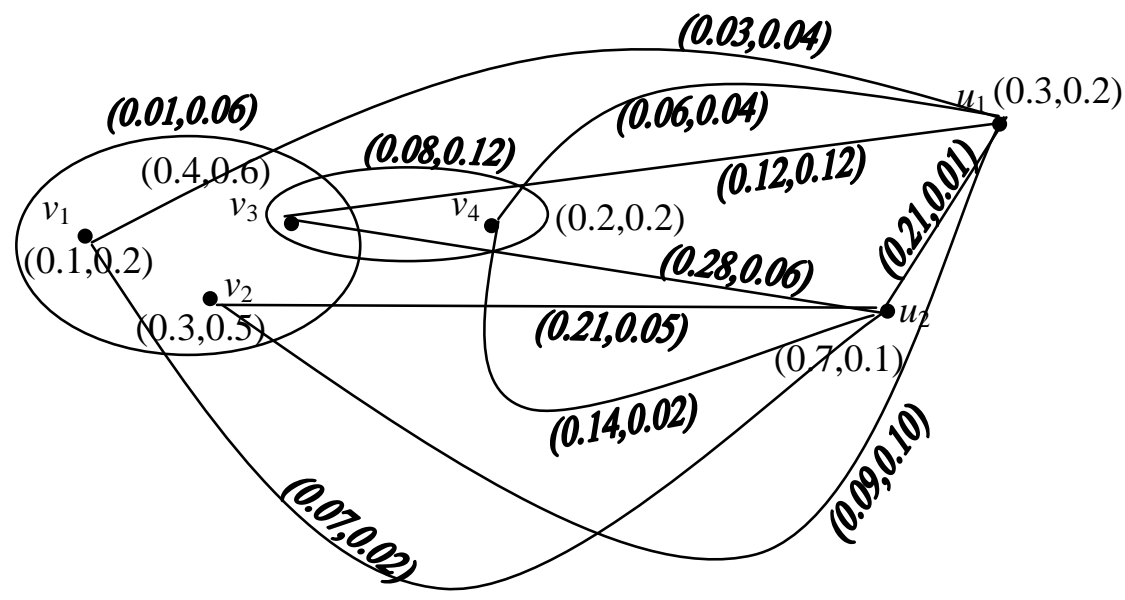

Fig 4: $G_{1}+G_{2}$ 


\section{Example 4.6}

For the operation intersection and ringsum we consider $V_{1}=\left\{v_{1}, v_{2}, v_{3}\right\}$ and $V_{2}=\left\{v_{1}, v_{2}, v_{3}\right\}$ and $V_{1} \mathrm{I} V_{2} \neq \varphi$
Theorem 4.7

Let $G_{1}=\left\langle V_{1}, E_{1}\right\rangle$ and $G_{2}=\left\langle V_{2}, E_{2}\right\rangle$ be two IFHGs. Then (i) $\overline{G_{1}+G_{2}} \cong \overline{G_{1}} \mathrm{U} \overline{G_{2}}$ (ii) $\overline{G_{1} U G_{2}} \cong \overline{G_{1}}+\overline{G_{2}}$

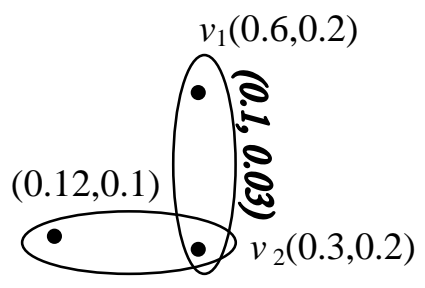

$v_{3}(0.4,0.5)$

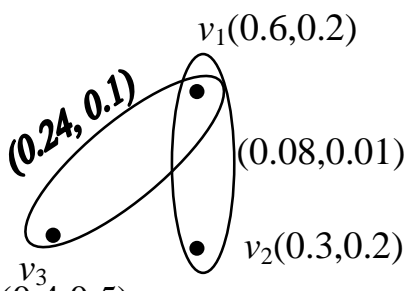

$(0.4,0.5)$

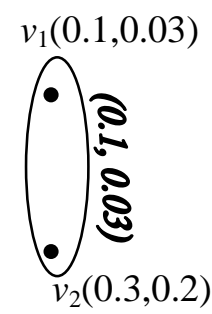

$(0.4,0.5)$

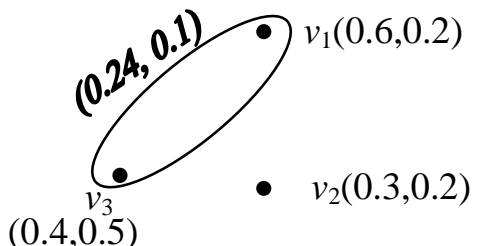

a)

b)

c)

d)

\section{Proof}

Consider the identity map $I: V_{1} \mathrm{U} V_{2} \rightarrow V_{1} \mathrm{U} V_{2}$.To prove (i), it is enough to prove

a) (i) $\left(\overline{\mu_{1}+\mu_{1}^{\prime}}\right)\left(v_{i}\right)=\left(\overline{\mu_{1}} \mathrm{U} \overline{\mu_{1}^{\prime}}\right)\left(v_{i}\right)$

(ii) $\left(\overline{\gamma_{1}+\gamma_{1}^{\prime}}\right)\left(v_{i}\right)=\left(\overline{\gamma_{1}} \mathrm{U} \overline{\gamma_{1}^{\prime}}\right)\left(v_{i}\right)$

(b) (i) $\left(\overline{\mu_{2}+\mu_{2}^{\prime}}\right)\left(v_{i}, v_{j}\right)=\left(\overline{\mu_{2}} \mathrm{U} \overline{\mu_{2}^{\prime}}\right)\left(v_{i}, v_{j}\right)$

(ii) $\left(\overline{\gamma_{2}+\gamma_{2}^{\prime}}\right)\left(v_{i}, v_{j}\right)=\left(\overline{\gamma_{2}} \mathrm{U} \overline{\gamma_{2}^{\prime}}\right)\left(v_{i}, v_{j}\right)$.

(a) (i) $\left(\overline{\mu_{1}+\mu_{1}^{\prime}}\right)\left(v_{i}\right)=\left(\mu_{1}+\mu_{1}^{\prime}\right)\left(v_{i}\right)$, by Definition 4.1

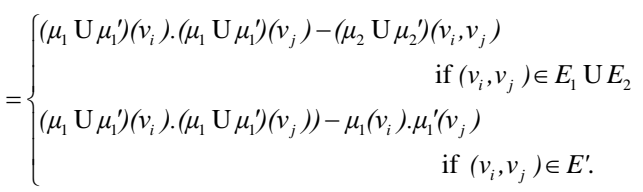

$=\left\{\begin{array}{l}\mu_{1}\left(v_{i}\right) \cdot \mu_{1}\left(v_{j}\right)-\mu_{2}\left(v_{i}, v_{j}\right) \quad \text { if }\left(v_{i}, v_{j}\right) \in E_{1} \\ \left.\mu_{1}^{\prime}\left(v_{i}\right) \cdot \mu_{1}^{\prime}\left(v_{j}\right)\right)-\mu_{2}^{\prime}\left(v_{i}, v_{j}\right) \quad \text { if }\left(v_{i}, v_{j}\right) \in E_{2} \\ \mu_{1}\left(v_{i}\right) \cdot \mu_{1}^{\prime}\left(v_{j}\right)-\mu_{1}\left(v_{i}\right) \cdot \mu_{1}^{\prime}\left(v_{j}\right) \text { if }\left(v_{i}, v_{j}\right) \in E^{\prime}\end{array}\right.$

$=\left\{\begin{array}{ll}\overline{\mu_{2}}\left(v_{i}, v_{j}\right) & \text { if }\left(v_{i}, v_{j}\right) \in E_{1} \\ \overline{\mu_{2}^{\prime}}\left(v_{i}, v_{j}\right) & \text { if }\left(v_{i}, v_{j}\right) \in E_{2} \\ 0 & \text { if }\left(v_{i}, v_{j}\right) \in E^{\prime}\end{array}=\left(\overline{\mu_{2}} \mathrm{U} \overline{\mu_{2}^{\prime}}\right)\left(v_{i}, v_{j}\right)\right.$.

$$
\begin{aligned}
& = \begin{cases}\mu_{1}\left(v_{i}\right) & \text { if } v_{i} \in V_{1} \\
\mu_{1}^{\prime}\left(v_{i}\right) & \text { if } v_{i} \in V_{2}\end{cases} \\
& =\left\{\begin{array}{ll}
\overline{\mu_{1}}\left(v_{i}\right) & \text { if } v_{i} \in V_{1} \\
\overline{\mu_{1}^{\prime}}\left(v_{i}\right) & \text { if } v_{i} \in V_{2}
\end{array}=\left(\overline{\mu_{1}} \mathrm{U} \overline{\mu_{1}^{\prime}}\right)\left(v_{i}\right) .\right.
\end{aligned}
$$

(ii) $\left(\overline{\gamma_{1}+\gamma_{1}^{\prime}}\right)\left(v_{i}\right)=\left(\gamma_{1}+\gamma_{1}^{\prime}\right)\left(v_{i}\right)$, by Definition 4.1

$=\left\{\begin{array}{l}\gamma_{1}\left(v_{i}\right) \text { if } v_{i} \in V_{1} \\ \gamma_{1}^{\prime}\left(v_{i}\right) \text { if } v_{i} \in V_{2}\end{array}= \begin{cases}\overline{\gamma_{1}}\left(v_{i}\right) & \text { if } v_{i} \in V_{1} \\ \overline{\gamma_{1}^{\prime}}\left(v_{i}\right) & \text { if } v_{i} \in V_{2}\end{cases}\right.$

(b)

(ii)

$\left(\overline{\gamma_{2}+\gamma_{2}^{\prime}}\right)\left(v_{i}, v_{j}\right)=\left(\gamma_{1}+\gamma_{1}^{\prime}\right)\left(v_{i}\right) \cdot\left(\gamma_{1}+\gamma_{1}^{\prime}\right)\left(v_{j}\right)-$ $\left(\gamma_{2}+\gamma_{2}^{\prime}\right)\left(v_{i}, v_{j}\right)$

$=\left\{\begin{array}{c}\left(\gamma_{1} \mathrm{U} \gamma_{1}^{\prime}\right)\left(v_{i}\right) \cdot\left(\gamma_{1} \mathrm{U} \gamma_{1}^{\prime}\right)\left(v_{j}\right)-\left(\gamma_{2} \mathrm{U} \gamma_{2}^{\prime}\right)\left(v_{i}, v_{j}\right) \\ \text { if }\left(v_{i}, v_{j}\right) \in E_{1} \mathrm{U} E_{2} \\ \left(\gamma_{1} \mathrm{U} \gamma_{1}^{\prime}\right)\left(v_{i}\right) \cdot\left(\gamma_{1} \mathrm{U} \gamma_{1}^{\prime}\right)\left(v_{j}\right)-\gamma_{1}\left(v_{i}\right) \cdot \gamma_{1}^{\prime}\left(v_{j}\right) \\ \text { if }\left(v_{i}, v_{j}\right) \in E^{\prime} .\end{array}\right.$

$=\left\{\begin{array}{l}\gamma_{1}\left(v_{i}\right) \cdot \gamma_{1}\left(v_{j}\right)-\gamma_{2}\left(v_{i}, v_{j}\right) \quad \text { if }\left(v_{i}, v_{j}\right) \in E_{1} \\ \gamma_{1}^{\prime}\left(v_{i}\right) \cdot \gamma_{1}^{\prime}\left(v_{j}\right)-\gamma_{2}^{\prime}\left(v_{i}, v_{j}\right) \quad \text { if }\left(v_{i}, v_{j}\right) \in E_{2} \\ \gamma_{1}\left(v_{i}\right) \cdot \gamma_{1}^{\prime}\left(v_{j}\right)-\gamma_{1}\left(v_{i}\right) \cdot \gamma_{1}^{\prime}\left(v_{j}\right) \quad \text { if }\left(v_{i}, v_{j}\right) \in E^{\prime}\end{array}\right.$

$=\left\{\begin{array}{lr}\overline{\gamma_{2}}\left(v_{i}, v_{j}\right) & \text { if }\left(v_{i}, v_{j}\right) \in E_{1} \\ \overline{\gamma_{2}^{\prime}}\left(v_{i}, v_{j}\right) & \text { if }\left(v_{i}, v_{j}\right) \in E_{2} \\ 0 & \text { if }\left(v_{i}, v_{j}\right) \in E^{\prime}\end{array}\right.$

(b) (i) $\begin{aligned} & \left(\overline{\mu_{2}+\mu_{2}^{\prime}}\right)\left(v_{i}, v_{j}\right)=\left(\mu_{1}+\mu_{1}^{\prime}\right)\left(v_{i}\right) \cdot\left(\mu_{1}+\mu_{1}^{\prime}\right)\left(v_{j}\right)- \\ & \left(\mu_{2}+\mu_{2}^{\prime}\right)\left(v_{i}, v_{j}\right)\end{aligned}$

$=\left(\overline{\gamma_{2}} \mathrm{U} \overline{\gamma_{2}^{\prime}}\right)\left(v_{i}, v_{j}\right)$. 
To prove (ii), it is enough to prove that

(a) (i) $\left(\overline{\mu_{1} \mathrm{U} \mu_{1}^{\prime}}\right)\left(v_{i}\right)=\left(\overline{\mu_{1}}+\overline{\mu_{1}^{\prime}}\right)\left(v_{i}\right)$

(ii) $\left(\overline{\gamma_{1} \mathrm{U} \gamma_{1}^{\prime}}\right)\left(v_{i}\right)=\left(\overline{\gamma_{1}}+\overline{\gamma_{1}^{\prime}}\right)\left(v_{i}\right)$

(b) (i) $\left(\overline{\mu_{2} \mathrm{U} \mu_{2}^{\prime}}\right)\left(v_{i}, v_{j}\right)=\left(\overline{\mu_{2}}+\overline{\mu_{2}^{\prime}}\right)\left(v_{i}, v_{j}\right)$

(ii) $\left(\overline{\gamma_{2} \mathrm{U} \gamma_{2}^{\prime}}\right)\left(v_{i}, v_{j}\right)=\left(\overline{\gamma_{2}}+\overline{\gamma_{2}^{\prime}}\right)\left(v_{i}, v_{j}\right)$.

Consider the identity map : $I: V_{1} \mathrm{U} V_{2} \rightarrow V_{1} \mathrm{U} V_{2}$.

(a) (i) $\left(\overline{\mu_{1} \mathrm{U} \mu_{1}^{\prime}}\right)\left(v_{i}\right)=\left(\mu_{1} \mathrm{U} \mu_{1}^{\prime}\right)\left(v_{i}\right)$

$=\left\{\begin{array}{l}\mu_{1}\left(v_{i}\right) \text { if } v_{i} \in V_{1} \\ \mu_{1}^{\prime}\left(v_{i}\right) \text { if } v_{i} \in V_{2}\end{array}= \begin{cases}\overline{\mu_{1}}\left(v_{i}\right) & \text { if } v_{i} \in V_{1} \\ \overline{\mu_{1}^{\prime}}\left(v_{i}\right) & \text { if } v_{i} \in V_{2}\end{cases}\right.$

$=\left(\overline{\mu_{1}} \mathrm{U} \overline{\mu_{1}^{\prime}}\right)\left(v_{i}\right)=\left(\overline{\mu_{1}}+\overline{\mu_{1}^{\prime}}\right)\left(v_{i}\right)$.

(a)(ii) $\left(\overline{\gamma_{1} \mathrm{U} \gamma_{1}^{\prime}}\right)\left(v_{i}\right)=\left(\gamma_{1} \mathrm{U} \gamma_{1}^{\prime}\right)\left(v_{i}\right)$

$=\left\{\begin{array}{ll}\gamma_{1}\left(v_{i}\right) & \text { if } v_{i} \in V_{1} \\ \gamma_{1}^{\prime}\left(v_{i}\right) & \text { if } v_{i} \in V_{2}\end{array}= \begin{cases}\overline{\gamma_{1}}\left(v_{i}\right) & \text { if } v_{i} \in V_{1} \\ \overline{\gamma_{1}^{\prime}}\left(v_{i}\right) & \text { if } v_{i} \in V_{2}\end{cases}\right.$

$=\left(\overline{\gamma_{1}} \mathrm{U} \overline{\gamma_{1}^{\prime}}\right)\left(v_{i}\right)=\left(\overline{\gamma_{1}}+\overline{\gamma_{1}^{\prime}}\right)\left(v_{i}\right)$.

(b)(i)

$\left(\overline{\mu_{2} \mathrm{U} \mu_{2}^{\prime}}\right)\left(v_{i}, v_{j}\right)=\left(\mu_{1} \mathrm{U} \mu_{1}^{\prime}\right)\left(v_{i}\right) \cdot\left(\mu_{1} \mathrm{U} \mu_{1}^{\prime}\right)\left(v_{j}\right)-$ $\left(\mu_{2} \mathrm{U} \mu_{2}^{\prime}\right)\left(v_{i}, v_{j}\right)$

$=\left\{\begin{array}{l}\mu_{1}\left(v_{i}\right) \cdot \mu_{1}\left(v_{j}\right)-\mu_{2}\left(v_{i}, v_{j}\right) \quad \text { if }\left(v_{i}, v_{j}\right) \in E_{1} \\ \mu_{1}^{\prime}\left(v_{i}\right) \cdot \mu_{1}^{\prime}\left(v_{j}\right)-\mu_{2}^{\prime}\left(v_{i}, v_{j}\right) \quad \text { if }\left(v_{i}, v_{j}\right) \in E_{2} \\ \mu_{1}\left(v_{i}\right) \cdot \mu_{1}^{\prime}\left(v_{j}\right)-0 \quad \text { if } v_{i} \in V_{1}, v_{j} \in V_{2}\end{array}\right.$

$=\left\{\begin{array}{l}\overline{\mu_{2}}\left(v_{i}, v_{j}\right) \quad \text { if }\left(v_{i}, v_{j}\right) \in E_{1} \\ \overline{\mu_{2}^{\prime}}\left(v_{i}, v_{j}\right) \quad \text { if }\left(v_{i}, v_{j}\right) \in E_{2} \\ \mu_{1}\left(v_{i}\right) \cdot \mu_{1}^{\prime}\left(v_{j}\right) \quad \text { if } v_{i} \in V_{1}, v_{j} \in V_{2}\end{array}\right.$

$=\left\{\begin{array}{l}\left(\overline{\mu_{2}} \mathrm{U} \overline{\mu_{2}^{\prime}}\right)\left(v_{i}, v_{j}\right) \quad \text { if }\left(v_{i}, v_{j}\right) \in E_{1} \text { or } E_{2} \\ \mu_{1}\left(v_{i}\right) \cdot \mu_{1}^{\prime}\left(v_{j}\right) \quad \text { if }\left(v_{i}, v_{j}\right) \in E^{\prime}\end{array}\right.$

$=\left(\overline{\mu_{2}}+\overline{\mu_{2}^{\prime}}\right)\left(v_{i}, v_{j}\right)$

(b)(ii)

$\left(\overline{\gamma_{2} \mathrm{U} \gamma_{2}^{\prime}}\right)\left(v_{i}, v_{j}\right)=\left(\gamma_{1} \mathrm{U} \gamma_{1}^{\prime}\right)\left(v_{i}\right) \cdot\left(\gamma_{1} \mathrm{U} \gamma_{1}^{\prime}\right)\left(v_{j}\right)$

$-\left(\gamma_{2} \mathrm{U} \gamma_{2}{ }^{\prime}\right)\left(v_{i}, v_{j}\right)$

$=\left\{\begin{array}{l}\gamma_{1}\left(v_{i}\right) \cdot \gamma_{1}\left(v_{j}\right)-\gamma_{2}\left(v_{i}, v_{j}\right) \quad \text { if }\left(v_{i}, v_{j}\right) \in E_{1} \\ \gamma_{1}^{\prime}\left(v_{i}\right) \cdot \gamma_{1}^{\prime}\left(v_{j}\right)-\gamma_{2}^{\prime}\left(v_{i}, v_{j}\right) \quad \text { if }\left(v_{i}, v_{j}\right) \in E_{2} \\ \gamma_{1}\left(v_{i}\right) \cdot \gamma_{1}^{\prime}\left(v_{j}\right)-0 \quad \text { if } v_{i} \in V_{1}, v_{j} \in V_{2}\end{array}\right.$ $=\left\{\begin{array}{l}\overline{\gamma_{2}}\left(v_{i}, v_{j}\right) \quad \text { if }\left(v_{i}, v_{j}\right) \in E_{1} \\ \overline{\gamma_{2}^{\prime}}\left(v_{i}, v_{j}\right) \quad \text { if }\left(v_{i}, v_{j}\right) \in E_{2} \\ \gamma_{1}\left(v_{i}\right) \cdot \gamma_{1}^{\prime}\left(v_{j}\right) \quad \text { if } v_{i} \in V_{1}, v_{j} \in V_{2}\end{array}\right.$

$=\left\{\begin{array}{l}\left(\overline{\gamma_{2}} \mathrm{U} \overline{\gamma_{2}^{\prime}}\right)\left(v_{i}, v_{j}\right) \quad \text { if }\left(v_{i}, v_{j}\right) \in E_{1} \text { or } E_{2} \\ \gamma_{1}\left(v_{i}\right) \cdot \gamma_{1}^{\prime}\left(v_{j}\right) \text { if } \quad\left(v_{i}, v_{j}\right) \in E^{\prime}\end{array}\right.$

$=\left(\overline{\gamma_{2}}+\overline{\gamma_{2}^{\prime}}\right)\left(v_{i}, v_{j}\right)$.

\section{Definition 4.8}

Let $G=G_{1} \times G_{2}=\left\langle V, E^{\prime \prime}\right\rangle$ be the Cartesian product of two graphs $G_{1}$ and $G_{2}$ where $V=V_{1} \times V_{2}$ and

$$
\begin{aligned}
E^{\prime \prime}=\left\{\left(u, u_{2}\right)\left(u, v_{2}\right): u \in V_{1}, u_{2} v_{2} \in E_{2}\right\} \mathrm{U} \\
\left\{\left(u_{1}, w\right)\left(v_{1}, w\right): w \in V_{2}, u_{1} v_{1} \in E_{1}\right\} .
\end{aligned}
$$

Then, the Cartesian product of IFHGs $G_{1}$ and $G_{2}$ is an IFHG defined by $G=G_{1} \times G_{2}=\left\langle V, E^{\prime \prime}\right\rangle$ where

(i)

$\left(\mu_{1} \times \mu_{1}{ }^{\prime}\right)\left(u_{1}, u_{2}\right)=\mu_{1}\left(u_{1}\right) \cdot \mu_{1}{ }^{\prime}\left(u_{2}\right)$ for every $\left(u_{1}, u_{2}\right) \in V$ and

$\left(\gamma_{1} \times \gamma_{1}^{\prime}\right)\left(u_{1}, u_{2}\right)=\gamma_{1}\left(u_{1}\right) \cdot \gamma_{1}^{\prime}\left(u_{2}\right)$ for every $\left(u_{1}, u_{2}\right) \in V$

(ii)

$$
\begin{aligned}
& \left(\mu_{2} \times \mu_{2}^{\prime}\right)\left(u, u_{2}\right)\left(u, v_{2}\right)=\mu_{1}(u) \cdot \mu_{2}\left(u_{2} v_{2}\right) \\
& \text { for every } u \in V_{1} \text {, and } u_{2} v_{2} \in E_{2} \\
& \left(\gamma_{2} \times \gamma_{2}^{\prime}\right)\left(u, u_{2}\right)\left(u, v_{2}\right)=\gamma_{1}(u) \cdot \gamma_{2}\left(u_{2} v_{2}\right) \\
& \text { for every } u \in V_{1} \text {, and } u_{2} v_{2} \in E_{2} \text { and }
\end{aligned}
$$

$\left(\mu_{2} \times \mu_{2}^{\prime}\right)\left(u_{1}, w\right)\left(v_{1}, w\right)=\mu_{1}(w) \cdot \mu_{2}\left(u_{1} v_{1}\right)$

for every $w \in V_{2}$, and $u_{1} v_{1} \in E_{1}$ $\left(\gamma_{2} \times \gamma_{2}^{\prime}\right)\left(u_{1}, w\right)\left(v_{1}, w\right)=\gamma_{1}(w) \cdot \gamma_{2}\left(u_{1} v_{1}\right)$

for every $w \in V_{2}$, and $u_{1} v_{1} \in E_{1}$.

\section{Example 4.9}

Let $V_{1}=\left\{v_{1}, v_{2}, v_{3}, v_{4}\right\}$ and $V_{2}=\left\{u_{1}, u_{2}\right\}$ such that $V_{1} \mathrm{I} V_{2}=\varphi$ 


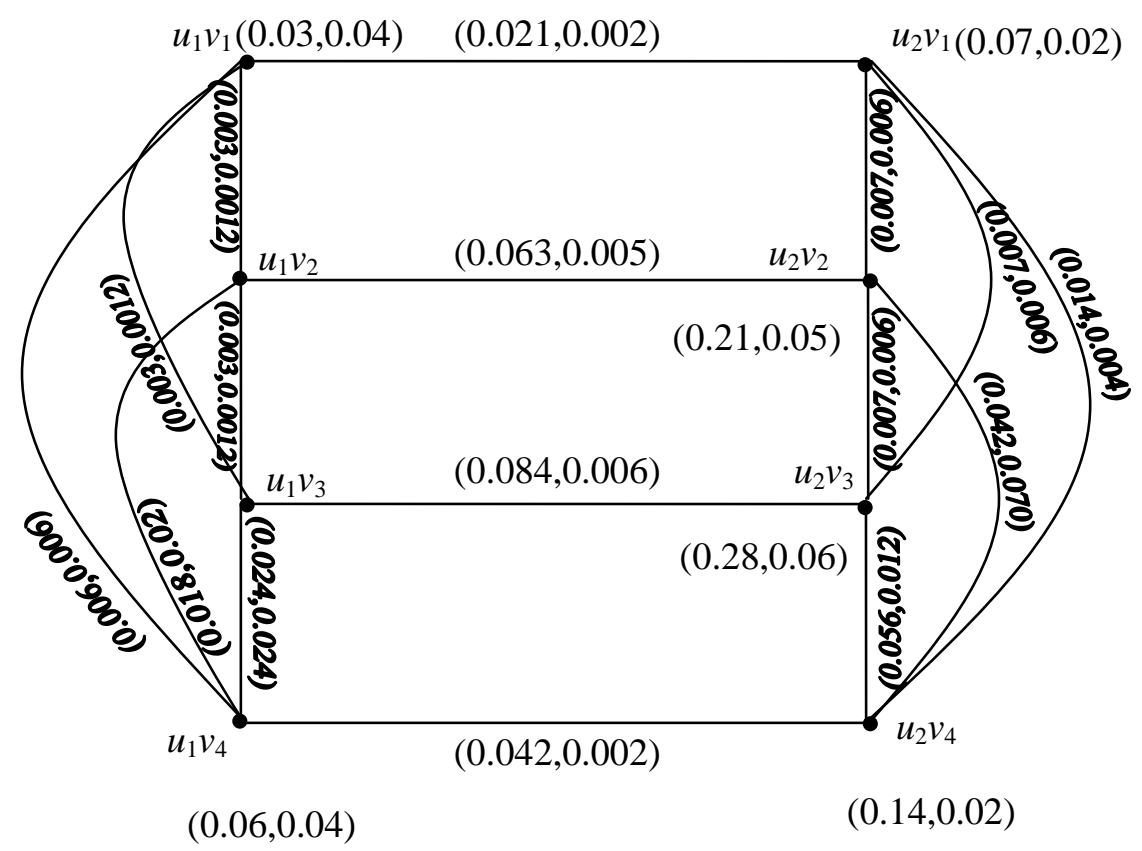

Fig 6: $G_{1} \times G_{2}$

\section{Definition 4.10}

Let $G=G_{1} \circ G_{2}=\left(V_{1} \times V_{2}, E\right)$ be the composition of two graphs $G_{1}$ and $G_{2}$,

where

$E=\left\{\left(u, u_{2}\right)\left(u, v_{2}\right): u \in V_{1}, u_{2} v_{2} \in E_{2}\right\} \mathrm{U}$

$\left\{\left(u_{1}, w\right)\left(v_{1}, w\right): w \in V_{2}, u_{1} v_{1} \in E_{1}\right\} \mathrm{U}$

$\left\{\left(u_{1}, u_{2}\right)\left(v_{1}, v_{2}\right): u_{1} v_{1} \in E_{1}, u_{2} \neq v_{2}\right\}$.

Then the composition of IFHGs $G_{1}$ and $G_{2}$, denoted by $G=G_{1} \mathrm{o} G_{2}$, is an IFHG defined by

(i) $\left(\mu_{1} \mathrm{o} \mu_{1}^{\prime}\right)\left(u_{1}, u_{2}\right)=\mu_{1}\left(u_{1}\right) \cdot \mu_{1}^{\prime}\left(u_{2}\right)$ for every $\left(u_{1}, u_{2}\right) \in V_{1} \times V_{2}$ and

$\left(\gamma_{1} \mathrm{o} \gamma_{1}^{\prime}\right)\left(u_{1}, u_{2}\right)=\gamma_{1}\left(u_{1}\right) \cdot \gamma_{1}^{\prime}\left(u_{2}\right)$ for every $\left(u_{1}, u_{2}\right) \in V_{1} \times V_{2}$

(ii) $\left(\mu_{2} \mathrm{o} \mu_{2}^{\prime}\right)\left(u, u_{2}\right)\left(u, v_{2}\right)=\mu_{1}(u) \cdot \mu_{2}\left(u_{2} v_{2}\right)$ for every $u \in V_{1}$,

$\left(\gamma_{2} \mathrm{o} \gamma_{2}^{\prime}\right)\left(u, u_{2}\right)\left(u, v_{2}\right)=\gamma_{1}(u) \cdot \gamma_{2}\left(u_{2} v_{2}\right)$ for every $u \in \mathrm{V}_{1}$, and $u_{2} v_{2} \in E_{2}$

$$
\left(\mu_{2} \mathrm{o} \mu_{2}^{\prime}\right)\left(u_{1}, w\right)\left(v_{1}, w\right)=\mu_{1}(w) \cdot \mu_{2}\left(u_{1} v_{1}\right)
$$

and for every $w \in V_{2}$, and $u_{1} v_{1} \in E_{1}$

$\left(\gamma_{2} \mathrm{o} \gamma_{2}^{\prime}\right)\left(u_{1}, w\right)\left(v_{1}, w\right)=\gamma_{1}(w) \cdot \gamma_{2}\left(u_{1} v_{1}\right)$

for every $w \in V_{2}$, and $u_{1} v_{1} \in E_{1}$ $\left(\mu_{2} \mathrm{o} \mu_{2}^{\prime}\right)\left(u_{1}, u_{2}\right)\left(v_{1}, v_{2}\right)=\mu_{1}^{\prime}\left(u_{2}\right) \cdot \mu_{1}^{\prime}\left(v_{2}\right) \cdot \mu_{2}\left(u_{1}, v_{1}\right)$ for every $\left(u_{1}, u_{2}\right)\left(v_{1}, v_{2}\right) \in E-E^{\prime \prime}$

and

$\left(\gamma_{2} \mathrm{o} \gamma_{2}^{\prime}\right)\left(u_{1}, u_{2}\right)\left(v_{1}, v_{2}\right)=\gamma_{1}^{\prime}\left(u_{2}\right) \cdot \gamma_{1}^{\prime}\left(v_{2}\right) \cdot \gamma_{2}\left(u_{1}, v_{1}\right)$

for every $\left(u_{1}, u_{2}\right),\left(v_{1}, v_{2}\right) \in E-E^{\prime \prime}$

where

$E^{\prime \prime}=\left\{\left(u, u_{2}\right)\left(u, v_{2}\right): u \in V_{1}\right.$, for every $\left.u_{2} v_{2} \in E_{2}\right\} \mathrm{U}$

$\left\{\left(u_{1}, w\right)\left(v_{1}, w\right): w \in V_{2}\right.$, for every $\left.u_{1} v_{1} \in E_{1}\right\}$.

\section{Theorem 4.9}

Let $G_{1}=\left\langle V_{1}, E_{1}\right\rangle$ and $G_{2}=\left\langle V_{2}, E_{2}\right\rangle$ be two strong IFHGs. Then, $G_{1} \circ G_{2}$ is a strong IFHG.

Proof

Let $G_{1} \circ G_{2}=G=\langle V, E\rangle$ where $V=V_{1} \times V_{2}$ and

$$
\begin{aligned}
& E=\left\{\left(u, u_{2}\right)\left(u, v_{2}\right): u \in V_{1}, u_{2} v_{2} \in E_{2}\right\} \mathrm{U} \\
& \left\{\left(u_{1}, w\right)\left(v_{1}, w\right): w \in V_{2}, u_{1} v_{1} \in E_{1}\right\} \mathrm{U} \\
& \quad\left\{\left(u_{1}, u_{2}\right)\left(v_{1}, v_{2}\right): u_{1} v_{1} \in E_{1}, u_{2} \neq v_{2}\right\} . \\
& \text { (i) } \mu_{2}\left(u, u_{2}\right)\left(u, v_{2}\right)=\mu_{1}(u) \cdot \mu_{2}\left(u_{2} v_{2}\right) \\
& =\mu_{1}(u) \cdot\left(\mu_{1}^{\prime}\left(u_{2}\right) \cdot \mu_{1}^{\prime}\left(v_{2}\right)\right) \text {, since } G_{2} \text { is strong }
\end{aligned}
$$


$=\mu_{1}(u) \cdot \mu_{1}^{\prime}\left(u_{2}\right) \cdot \mu_{1}(u) \cdot \mu_{1}^{\prime}\left(v_{2}\right)$

$=\left(\mu_{1} \mathrm{o} \mu_{1}^{\prime}\right)\left(u, u_{2}\right) \cdot\left(\mu_{1} \mathrm{o} \mu_{1}^{\prime}\right)\left(u, v_{2}\right)$.

$\gamma_{2}\left(u, u_{2}\right)\left(u, v_{2}\right)=\gamma_{1}(u) \cdot \gamma_{2}\left(u_{2} v_{2}\right)$

$=\gamma_{1}(u) \cdot\left(\gamma_{1}^{\prime}\left(u_{2}\right) \cdot \gamma_{1}^{\prime}\left(v_{2}\right)\right)$, since $G_{2}$ is strong

$=\gamma_{1}(u) \cdot \gamma_{1}^{\prime}\left(u_{2}\right) \cdot \gamma_{1}(u) \cdot \gamma_{1}^{\prime}\left(v_{2}\right)$

$=\left(\gamma_{1} \mathrm{o} \gamma_{1}^{\prime}\right)\left(u, u_{2}\right) \cdot\left(\gamma_{1} \mathrm{o} \gamma_{1}^{\prime}\right)\left(u, v_{2}\right)$.

(ii) $\mu_{2}\left(\left(u_{1}, w\right)\left(v_{1}, w\right)\right)=\mu_{1}^{\prime}(w) \cdot \mu_{2}\left(u_{1}, v_{1}\right)$

$=\mu_{1}^{\prime}(w) \cdot\left(\mu_{1}\left(u_{1}\right) \cdot \mu_{1}\left(v_{1}\right)\right)$, since $G_{1}$ is strong

$=\mu_{1}^{\prime}(w) \cdot \mu_{1}\left(v_{1}\right) \cdot \mu_{1}^{\prime}(w) \cdot \mu_{1}\left(v_{1}\right)$

$=\left(\mu_{1} \mathrm{o} \mu_{1}^{\prime}\right)\left(u_{1}, w\right) \cdot\left(\mu_{1} \mathrm{o} \mu_{1}^{\prime}\right)\left(v_{1}, w\right)$.

$\gamma_{1}\left(\left(u_{1}, w\right)\left(v_{1}, w\right)\right)=\gamma_{1}^{\prime}(w) \cdot \gamma_{2}\left(u_{1}, v_{1}\right)$

$=\gamma_{1}^{\prime}(w) \cdot\left(\gamma_{1}\left(u_{1}\right) \cdot \gamma_{1}\left(v_{1}\right)\right)$, since $G_{1}$ is strong $=\gamma_{1}^{\prime}(w) \cdot \gamma_{1}\left(v_{1}\right) \cdot \gamma_{1}^{\prime}(w) \cdot \gamma_{1}\left(v_{1}\right)=\left(\gamma_{1} \mathbf{0} \gamma_{1}^{\prime}\right)\left(u_{1}, w\right) \cdot\left(\gamma_{1} \mathbf{0} \gamma_{1}^{\prime}\right)\left(v_{1}, w\right)$

(iii) $\mu_{2}\left(u_{1}, u_{2}\right)\left(v_{1}, v_{2}\right)=\mu_{2}\left(u_{1}, v_{1}\right) \cdot \mu_{1}^{\prime}\left(u_{2}\right) \cdot \mu_{1}^{\prime}\left(v_{2}\right)$

$=\left(\mu_{1}\left(u_{1}\right) \cdot \mu_{1}\left(v_{1}\right)\right) \cdot \mu_{1}^{\prime}\left(u_{2}\right) \cdot \mu_{1}^{\prime}\left(v_{2}\right), \quad G_{1}$ is strong

$=\mu_{1}\left(u_{1}\right) \cdot \mu_{1}^{\prime}\left(u_{2}\right) \cdot \mu_{1}\left(v_{1}\right) \cdot \mu_{1}^{\prime}\left(v_{2}\right)$

$=\left(\mu_{1} \mathrm{o} \mu_{1}^{\prime}\right)\left(u_{1}, u_{2}\right) \cdot\left(\mu_{1} \mathrm{o} \mu_{1}^{\prime}\right)\left(v_{1}, v_{2}\right)$.

$\gamma_{2}\left(u_{1}, u_{2}\right)\left(v_{1}, v_{2}\right)=\gamma_{2}\left(u_{1}, v_{1}\right) \cdot \gamma_{1}^{\prime}\left(u_{2}\right) \cdot \gamma_{1}^{\prime}\left(v_{2}\right)$

$=\left(\gamma_{1}\left(u_{1}\right) \cdot \gamma_{1}\left(v_{1}\right)\right) \cdot \gamma_{1}^{\prime}\left(u_{2}\right) \cdot \gamma_{1}^{\prime}\left(v_{2}\right), \quad G_{1}$ is strong

$=\left(\gamma_{1}\left(u_{1}\right) \cdot \gamma_{1}^{\prime}\left(u_{2}\right)\right) \cdot \gamma_{1}\left(v_{1}\right) \cdot \gamma_{1}^{\prime}\left(v_{2}\right)$

$=\left(\gamma_{1} \mathrm{o} \gamma_{1}^{\prime}\right)\left(u_{1}, u_{2}\right) \cdot\left(\gamma_{1} \mathrm{o} \gamma_{1}^{\prime}\right)\left(v_{1}, v_{2}\right)$.

From (i), (ii), (iii), $G$ is a strong IFHG.

Example 4.11

Let Let $V_{1}=\left\{v_{1}, v_{2}, v_{3}, v_{4}\right\}$ and $V_{2}=\left\{u_{1}, u_{2}\right\}$ such that $V_{1} \mathrm{I} \quad V_{2}=\varphi$

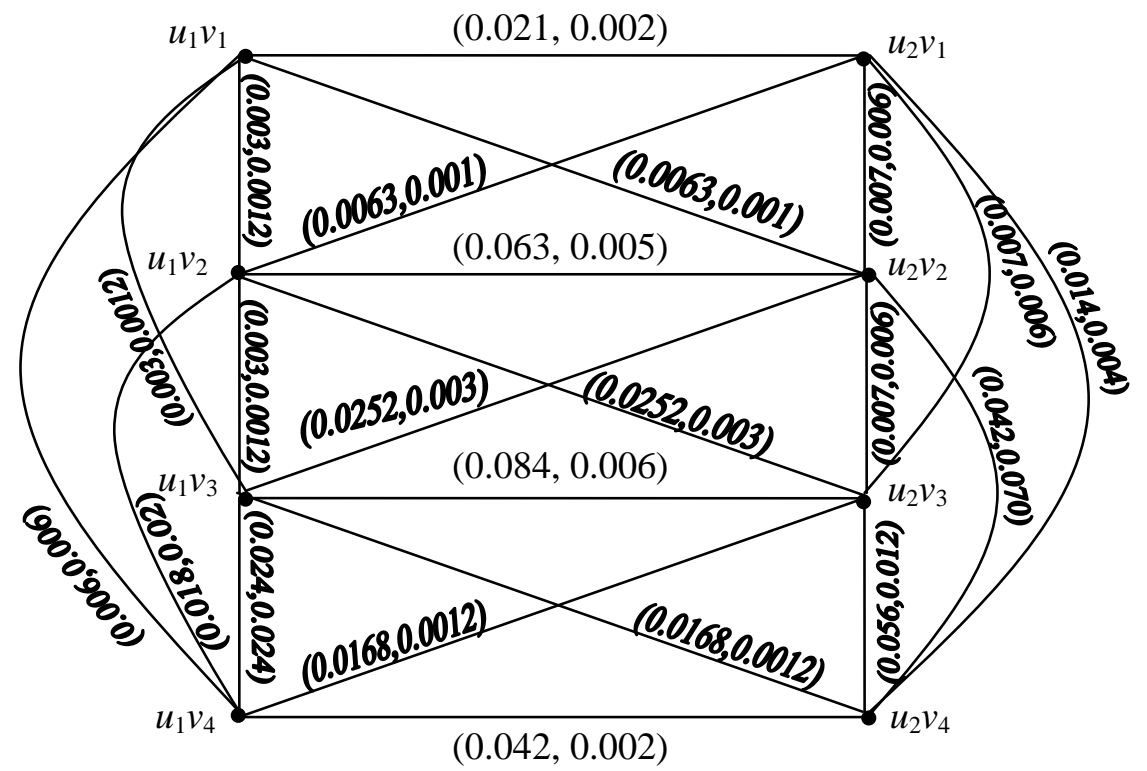

Fig 7: $G_{1} \circ G_{2}$

\section{CONCLUSION}

In this paper, operations like complement, join, union, intersection, ringsum, Cartesian product, composition on IFHGs are defined. Currently, the authors are working on existing clustering techniques. Further, it is proposed to apply the properties of IFHGs to develop a new clustering algorithm and the same may be checked with a numerical dataset 


\section{REFERENCES}

[1] K.T.Atanassov, Intuitionistic fuzzy sets, Fuzzy Sets and Systems 20, (1986), 87-96.

[2] K.T.Atanassov, More on intuitionistic fuzzy sets, Fuzzy Sets and Systems 33, (1989), 37-46.

[3] K.T.Atanassov, New operations defined over the intuitionistic fuzzy sets, Fuzzy Sets and Systems 61, (1994), 137-142.

[4] K.T.Atanassov, Remarks on the intuitionistic fuzzy setsIII, Fuzzy Sets and Systems 75, (1995), 401-402.

[5] S.Thilagavathi, R.Parvathi and M.G.Karunambigai, Operations on Intuitionistic fuzzy graphs II,
Developments in fuzzy sets, intuitionistic fuzzy sets, generalized nets and related topics 1, (2008), 319-331.

[6] R.Parvathi, M.G.Karunambigai and K.T.Atanassov Operations on Intuitionistic fuzzy graphs, Fuzzy Systems, 2009, Fuzz-IEEE 2009. IEEE international conference 1396-1401.

[7] S.Thilagavathi, R.Parvathi and M.G.Karunambigai, Intuitionistic fuzzy hypergraphs, J.Cybernetics and Information Technologies 9(2), (2009), 46-53, Sofia.

[8] M.Akram and W.A.Dudek Intuitionistic fuzzy hypergraphs with applications, Information Sciences, 2012 